\title{
Sonic hedgehog is expressed in human brain arteriovenous malformations and induces arteriovenous malformations in vivo
}

Journal of Cerebral Blood Flow \& Metabolism $0(0) 1-12$ (C) The Author(s) 2020 Article reuse guidelines: sagepub.com/journals-permissions DOI: $10.1177 / 0271678 \times 209 / 2405$ journals.sagepub.com/home/jcbfm @SAGE

\author{
Igor Giarretta', Carmelo L Sturiale ${ }^{2}$, Ilaria Gatto', \\ Simone Pacioni ${ }^{2}$, Eleonora Gaetani', Angelo Porfidia', \\ Alfredo Puca ${ }^{2}$, Ivana Palucci ${ }^{3}$, Paolo Tondi', Alessandro Olivi', \\ Roberto Pallini ${ }^{2}$ and Roberto Pola ${ }^{1,4}$ (1)
}

\begin{abstract}
Abnormalities in arterial versus venous endothelial cell identity and dysregulation of angiogenesis are deemed important in the pathophysiology of brain arteriovenous malformations (AVMs). The Sonic hedgehog (Shh) pathway is crucial for both angiogenesis and arterial versus venous differentiation of endothelial cells, through its dual role on the vascular endothelial growth factor/Notch signaling and the nuclear orphan receptor COUP-TFII. In this study, we show that Shh, Glil (the main transcription factor of the Shh pathway), and COUP-TFII (a target of the non-canonical Shh pathway) are aberrantly expressed in human brain AVMs. We also show that implantation of pellets containing Shh in the cornea of Efnb2/LacZ mice induces growth of distinct arteries and veins, interconnected by complex sets of arteriovenous shunts, without an interposed capillary bed, as seen in AVMs. We also demonstrate that injection in the rat brain of a plasmid containing the human Shh gene induces the growth of tangles of tortuous and dilated vessels, in part positive and in part negative for the arterial marker $\alpha$ SMA, with direct connections between $\alpha$ SMA-positive and -negative vessels. In summary, we show that the Shh pathway is active in human brain AVMs and that Shh-induced angiogenesis has characteristics reminiscent of those seen in AVMs in humans.
\end{abstract}

\section{Keywords}

Arteriovenous malformation, Sonic hedgehog, angiogenesis, VEGF, growth factors

Received 10 September 2019; Revised 18 February 2020; Accepted 19 February 2020

\section{Introduction}

Brain arteriovenous malformations (AVMs) are abnormalities of the intracranial vessels, with direct connections between the arterial and venous systems in the absence of an intervening capillary bed. AVMs can reach considerable size and undergo rupture and are the main non-traumatic cause of cerebral hemorrhage in young people. ${ }^{1}$

Etiology and pathogenesis of brain AVMs remain poorly understood, hindering the development of efficacious therapeutic strategies. Current theories propose that abnormalities in arterial versus venous identity of endothelial cells (ECs) are important for AVM formation. Indeed, AVM nidi co-express both arterial and
'Department of Medicine, Fondazione Policlinico Universitario A. Gemelli IRCCS, Università Cattolica del Sacro Cuore, Rome, Italy ${ }^{2}$ Division of Neurosurgery, Fondazione Policlinico Universitario A. Gemelli IRCCS, Università Cattolica del Sacro Cuore, Rome, Italy ${ }^{3}$ Istitute of Microbiology, Fondazione Policlinico Universitario A. Gemelli IRCCS, Università Cattolica del Sacro Cuore, Rome, Italy ${ }^{4}$ Division of Cardiovascular Research, St. Elizabeth's Medical Center, Boston, MA, USA

\section{Corresponding author:}

Roberto Pola, Istituto di Medicina Interna e Geriatria, Policlinico Universitario Agostino Gemelli, L.go A. Gemelli 8, 00168 Rome, Italy. Email: roberto.pola@unicatt.it 
venous markers, suggesting that AVMs consist of vessels that have undergone inadequate maturation and are not terminally differentiated. ${ }^{2}$ Nonetheless, it is known that AVMs can form de novo during childhood and/or adulthood as result of aberrant angiogenesis, arteriogenesis, and vasculogenesis. ${ }^{3-6}$

The Sonic hedgehog (Shh) signaling pathway is crucial for arterial versus venous differentiation of ECs. ${ }^{7}$ Studies in zebrafish have shown that embryos lacking Shh fail to express vascular endothelial growth factor (VEGF) within the somites and are unable to establish arterial identity in the dorsal aorta. ${ }^{8}$ Conversely, microinjection of messenger RNA encoding Shh in zebrafish embryos leads to a switch from a venous to an arterial fate in the posterior cardinal vein. ${ }^{8}$ It is now accepted that Shh regulates VEGF expression and acts upstream of Notch to induce arterial differentiation of ECs also in mammals and in postnatal life. ${ }^{8,9}$ VEGF induction by Shh occurs through the activation of the canonical Shh signaling pathway, with the involvement of the glioma-associated oncogene homolog (GLI) family of transcription factors. ${ }^{10}$ On the other hand, Shh has also the ability to activate COUP-TFII, through a GLIindependent non-canonical mechanism, since the COUP-TFII promoter contains a Shh response element that mediates Shh-induced activity. ${ }^{11}$ Importantly, COUP-TFII is a down-regulator of Notch and is specifically expressed in venous, but not arterial, endothelium. ${ }^{12}$

$\mathrm{Shh}$ is also a major regulator of angiogenesis, arteriogenesis, and vasculogenesis and has the ability to upregulate various families of angiogenic growth factors, including three different isoforms of VEGF-A, VEGF-D, angiopoietins, insulin-like growth factor-1, fibroblast growth factor, and stromal-derived factor$1 \alpha .{ }^{13-16}$ Interestingly, Shh-induced angiogenesis has a distinctive morphology characterized by enlarged and apparently poorly organized tubular structures. ${ }^{13}$

In this study, we evaluated the expression of Shh, its main transcription factor Gli1, and its non-canonical target COUP-TFII, in human brain AVMs. We also tested the hypothesis that Shh has the ability to induce the formation of AVMs in vivo.

\section{Materials and methods}

The data that support the findings of this study are available from the corresponding author upon reasonable request.

\section{Shh pathway expression in human brain AVMs}

Analyses were performed on eight archival paraffinembedded specimens of human brain AVMs surgically treated at the Institute of Neurosurgery, Università
Cattolica del Sacro Cuore of Rome, Italy. Specimens of pediatric patients and patients with hereditary hemorrhagic telangiectasia were excluded from the study. Archival paraffin-embedded specimens of human brain derived from epilepsy surgery $(n=5)$ were used as controls. The clinical characteristics of patients and controls are available in the Supplementary Tables 1 and 2. Primary antibodies were: rabbit polyclonal anti-Shh, anti-Gli1, and anti-COUP-TFII (Origene, Rockville, MD, USA). Secondary antibody was CFR Antipolivalent HRP Polymer (Scytec Laboratories, Logan, UT, USA). Protein expression was visualized using the avidin-biotin-peroxidase complex method (Dako AutoStainer, Carpinteria, CA, USA). Specimens of human kidney cancer, brain, and tonsil were used as positive controls for Shh, Glil, and COUP-TFII, respectively. The study was in compliance with the Declaration of Helsinki and was approved by the Ethics Committee of the Università Cattolica del Sacro Cuore, Rome, Italy. Due to the nature of the study, which only used archival specimens, informed consent from patients was not needed.

\section{Shh-induced angiogenesis in murine cornea}

Efnb2/LacZ heterozygous mice (8-12 weeks of age) $(n=18)$ (Jackson Laboratories, Bar Harbor, ME, USA) were used for these experiments. These mice carry a non-disruptive insertion of the LacZ reporter gene under the control of the Efnb2 promoter. LacZ expression corresponds to Efnb2 expression and, since Efnb2 is exclusively expressed in the arterial endothelium and is absent in the venous endothelium, allows distinguishing between arteries and veins. ${ }^{17}$ Corneal micropockets were created as described. ${ }^{13,18,19}$ Pellets containing Shh $0.5 \mu \mathrm{g}$, VEGF $0.5 \mu \mathrm{g}$, or PBS (empty pellets) were implanted in corneal micropockets. A total of 18 mice were used and, in each animal, both corneas were used, in order to have 12 corneas implanted with Shh pellets, 12 corneas implanted with VEGF pellets, and 12 corneas implanted with PBS pellets. Corneas were assigned randomly to each group of pellets. Six days later, mice were euthanized, eyes were enucleated, fixed, and used for whole mount immunofluorescent (six corneas per group) or histochemical analyses (six corneas per group). For immunofluorescent studies, corneal hemispheres were prepared under a dissecting microscope and incubated with anti-CD31 (BD Pharmingen, Franklin Lakes, NJ, USA) and anti$\beta$-gal antibodies (Abcam, Cambridge, UK). FITC and/ or Cy3-conjugated secondary antibodies (Thermo Fisher Scientific, Waltham, MA, USA) were used for fluorescent detection. For histochemical staining, corneas were embedded in paraffin, cut in cross-sections, and stained using the same anti-mouse CD31 antibody 
described above followed by a biotinylated goat antirabbit immunoglobulin secondary antibody (Abcam, Cambridge, UK). X-gal staining was performed as established. ${ }^{20}$ Corneal angiogenesis was quantified as described previously. ${ }^{13,18,19}$ Briefly, for each cornea, a minimum of five sections were observed under the microscope, in order to assess vessel length, circumferential extent of neovascularity, lumen diameter, arteries/veins ratio, mean length and diameter of arteries, and mean length and diameter of veins. All quantifications were performed in a blinded fashion. The experiments were conducted at the Division of Cardiovascular Research of St. Elizabeth's Medical Center, Boston, MA, in accordance with the American Association for the Accreditation of Laboratory Animal Care (AAALAC) policies and were approved by the Ethics Committee of the St. Elizabeth's Medical Center. Results are reported in compliance with the ARRIVE (Animal Research: Reporting in Vivo Experiments) guidelines.

\section{Shh-induced angiogenesis in rat brain}

To test whether the activation of the Hegdhog (Hh) pathway in the brain induces angiogenic growth reminiscent of AVM, we used the plasmid human Shh (phShh), which is a pCMV-Script vector containing the human Shh cDNA that we have used previously to induce functional activation of the $\mathrm{Hh}$ pathway in experimental models of myocardial ischemia and peripheral limb ischemia in mice, rats, and pigs. ${ }^{21-24}$ The animals used for these experiments were Wistar rats (average weight $250 \mathrm{~g}$ ). They received an intracerebral injection of $300 \mu \mathrm{g}$ phShh, resuspended in $50 \mu 1$ PBS. The amount of phShh to be injected was calculated on the basis of the relative mass of brain compared to heart and skeletal muscle in rats and the dose of phShh that we used in our previous experiments. ${ }^{21-24}$ The injections were performed under general anesthesia, as follows: animal skulls were immobilized in a stereotactic head frame, a burr hole was made $2 \mathrm{~mm}$ right of the midline and $1 \mathrm{~mm}$ anterior to the coronal suture, and the solution containing the phShh was slowly injected using the tip of a $100-\mu 1$ Hamilton microsyringe placed at a depth of $5 \mathrm{~mm}$ from the dura. The injection of phShh was performed unilaterally in the right brain hemisphere. Injection of empty plasmid $(300 \mu \mathrm{g})$ was performed in the left hemispheres of the same rat brains, which served as internal controls. This procedure has been described previously and allows the operator to selectively inject the plasmid at the level of the corpus striatum. ${ }^{25,26}$ A total of five rats were used. The calculation of the sample size was based on an estimated incidence of AVMs of $90 \%$ in rat brain hemispheres injected with phShh and of $10 \%$ in hemispheres injected with the empty plasmid. To achieve $80 \%$ power at the $5 \%$ significance level, we calculated a required sample size of at least five rats per group. In compliance with the $3 \mathrm{R}$ principles - Replace, Reduce and Refine - for the ethical use of animals in medicine testing, both cerebral hemispheres of each animal were used (the right hemisphere for the injection of phShh and the left hemisphere for the injection of the empty plasmid).

After the intracerebral injections, animals were kept under pathogen-free conditions in positive-pressure cabinets (Tecniplast Gazzada, Varese, Italy) and observed daily for neurological signs. Body weight was monitored weekly. Two weeks after injection, rats were deeply anesthetized and transcardially perfused with $0.1 \mathrm{M}$ PBS ( $\mathrm{pH} 7.4$ ), then treated with $4 \%$ paraformaldehyde in $0.1 \mathrm{M}$ PBS. Brains were removed, stored, serially cryostat sectioned, and used for immunodetection of CD31, using a polyclonal mouse antiCD31 antibody (BD Bioscience, Franklin Lakes, NJ, USA), and $\alpha$-smooth muscle actin ( $\alpha$ SMA), using a polyclonal goat anti- $\alpha$ SMA antibody (Abcam, Cambridge, UK). Secondary antibodies were: Alexa Fluor 647 donkey anti-mouse (1:500) (Abcam, Cambridge, UK) and Cy3 donkey anti-goat (1:200) (EMD Millipore, Billerica, MA, USA). For the detection of COUP-TFII expression in brain vasculature, sections were incubated with lectin from Lycopersicon esculentum (tomato) biotin conjugate (1:500) (SigmaAldrich, St. Louis, MO, USA) together with a rabbit polyclonal anti-COUP-TFII antibody (Origene, Rockville, MD, USA). The secondary antibody for the detection of COUP-TFII was an Alexa Fluor 647 donkey anti-rabbit (1:100) (Abcam, Cambridge, UK). Before mounting, slices were incubated with phosphate buffer containing 4',6-diamidino-2-phenylindole (DAPI) (1:4000) (Sigma-Aldrich, St. Louis, MO, USA). For each rat hemisphere, a minimum of five brain sections were observed under a laser confocal microscope (SP5, Leica, Wetzlar. Germany). The number of $\alpha$ SMA-positive and $\alpha$ SMA-negative vessels, the mean lumen diameter of $\alpha$ SMA-positive and $\alpha$ SMA-negative vessels, and the number of CD31positive vessels in the area surrounding the neovascular tangle were assessed using a dedicated Leica Application Suite X software. The analysis of histological sections was performed in a blinded fashion by two independent operators unaware of the specimen origin.

To determine which brain cells were transfected by our plasmid, we inserted the DsRedCherry reporter gene in the pCMV-Script plasmid, upon removal of the Shh gene sequence using the BamHI-NotI restriction enzyme (New England Biolab, Beverly, MA, USA). Then, the pCMV-DsRedCherry $(300 \mu \mathrm{g})$ was injected in the left brain hemisphere of four Wistar 
rats, using the same stereotactic methodology described above. Two weeks later, rats were sacrificed and brains were harvested, stored, serially cryostat sectioned, and processed for mCherry detection using a laser confocal microscope (SP5, Leica, Wetzlar, Germany). To determine whether mCherry was expressed in astrocytes or neuronal cells, brain sections were incubated with a polyclonal rabbit anti-Glial Fibrillary Acidic Protein (GFAP) (1:1000; Dako-Agilent, Santa Clara, CA, USA) or a polyclonal rabbit Synaptophysin antibody (1:250; Invitrogen Corporation, Carlsbad, CA, USA), using the same protocols described above. Slices were then incubated with Alexa Fluor 488-conjugated donkey anti rabbit secondary antibodies (1:400; Thermo Fisher Scientific, Waltham, MA).

The experiments were performed at the Università Cattolica del Sacro Cuore, Rome, Italy, according to the Directive 2010/63/EU of the European Parliament and of the Council of 22 September 2010 on the protection of animals used for scientific purposes and were approved by the Ethics Committee of the Fondazione Policlinico Universitario A. Gemelli IRCCS. Results are reported in compliance with the ARRIVE (Animal Research: Reporting in Vivo Experiments) guidelines.

\section{Statistical analysis}

All results are reported as mean \pm SD. Differences between groups were analyzed by paired t-test and considered statistically significant for $\mathrm{P}<0.05$. Calculations were performed with Prism 7 (GraphPad Software; www.graphpad.com/scientific-software/ prism/) and IBM SPSS Statistics for windows, Version 20.0, released 2011 (IBM Corp; www.ibm. com/analytics/it/it/technology/spss/).

\section{Results}

\section{Aberrant expression of Shh signaling in human brain AVMs}

The results of immunohistochemical analysis of surgically resected human brain AVMs, compared to normal human brain, are presented in Figure 1. We found distinct Shh expression in the endothelial layer of the AVM vessels. Also Glil was expressed in the endothelium of human brain AVMs. COUP-TFII was expressed both in the endothelium and the vascular wall of the human brain AVMs that we analyzed. No Shh, Gli1, or COUP-TFII-positive staining was found in normal human brain vessels.

\section{Shh-induced angiogenesis has AVM-like features in vivo}

The morphology of neoangiogenesis induced by Shh was strikingly different from that induced by VEGF in the murine cornea. In particular, Shh-induced neovasculature consisted of large, branching vessels that grew directly from the limbus vessels and extended to and surrounded the pellet at the apex of the new vessel growth (Figure 2(a)). In contrast, VEGF-induced angiogenesis mainly consisted of shorter vessels (unable to reach the pellet), uniform in size and length, distributed along the corneal circumference in

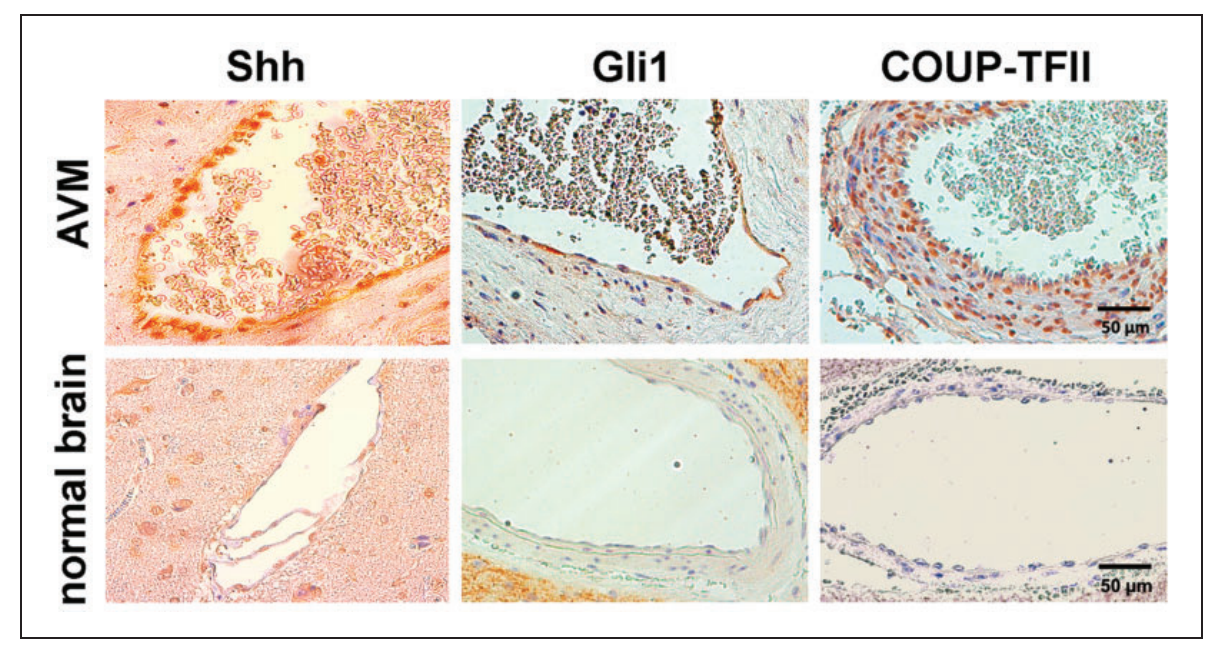

Figure I. Immunohistochemical analysis of Shh pathway expression in human brain AVMs. Representative images of human brain AVMs and control brain vessels. Cells in the intimal layer of AVMs are strongly immunopositive for Shh and Glil. COUP-TFII immunopositive cells are detectable in the intimal and medial layers of AVMs. Control brain vessels are negative in terms of Shh, Glil, and COUP-TFIl expression.

Shh: Sonic hedgehog. 
an orderly fashion (Figure 2(b)). In the setting of Shhinduced neonagiogenesis, it was possible to identify Efnb2-positive vessels originating directly from the main limbus artery, as well as Efnb2-negative vessels directly connected to the main limbus vein (Figure 2 (a)). In contrast, VEGF-induced angiogenesis mainly consisted of Efnb2-positive vessels (Figure 2(b)). Histological analysis of corneal cross sections confirmed the concomitant presence of arteries and veins in the setting of Shh-induced corneal angiogenesis (Figure 2(c)). Importantly, many Shh-induced vessels exhibited branching and created complex vascular trees with direct connections between arteries and veins, without the presence of an interposed capillary bed, as seen in AVMs (Figure 2(d)).

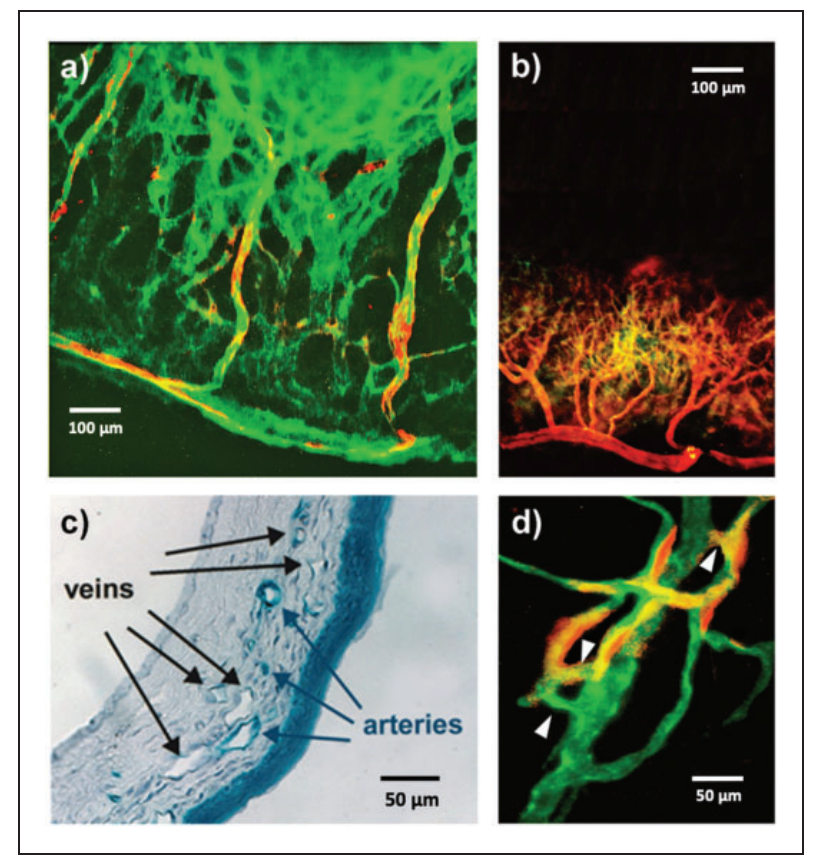

Figure 2. Characterization of the arterial and venous phenotype of Shh-induced corneal neovessels. (a) When pellets containing Shh are implanted in the corneal of Efnb2/LacZ mice, the resulting angiogenic process consists of large Efnb2-positive arterial vessels (red/yellow staining) that grow directly from the main limbus artery toward the pellet and large and tortuous Efnb2-negative, CD3I-positive veins (green staining) directly connected to the main limbus vein. (b) When pellets containing VEGF are implanted in the cornea of Efnb2/LacZ mice, the resulting angiogenic process consists of short Efnb2-positive arterial vessels (red/yellow staining) that grow directly from the main limbus artery and are orderly distributed along the corneal circumference. (c) X-gal staining of the corneas of Efnb2/LacZ mice implanted with Shh-containing pellets shows the concomitant presence of $X$-gal positive arteries (blue staining) and $X$-gal negative veins. (d) In the corneas of Efnb2/LacZ mice, Shhinduced angiogenesis is characterized by the presence of direct connections (white arrowheads) between Efnb2-positive arteries (red/yellow staining) and Efnb2-negative, CD3I-positive veins (green staining).
Corneal angiogenesis induced by Shh and VEGF was quantified in terms of vessel length, circumferential extent of neovascularity, lumen diameter, and arteries/ veins ratio (Figure 3(a)). The average length of Shh-induced neovessels was significantly greater than that of VEGF-induced vessels $(1.2 \pm 0.3$ versus $0.6 \pm 0.1 \mathrm{~mm} ; \mathrm{P}<0.0001)$. Increased lumen diameter was also seen in Shh- versus VEGF-induced neovessels $(32.1 \pm 5.5$ versus $7.8 \pm 1.5 \mu \mathrm{m} ; \mathrm{P}<0.0001)$. Also the circumferential extent of Shh-induced neovasculature was increased compared with VEGF (184.2 \pm 2.8

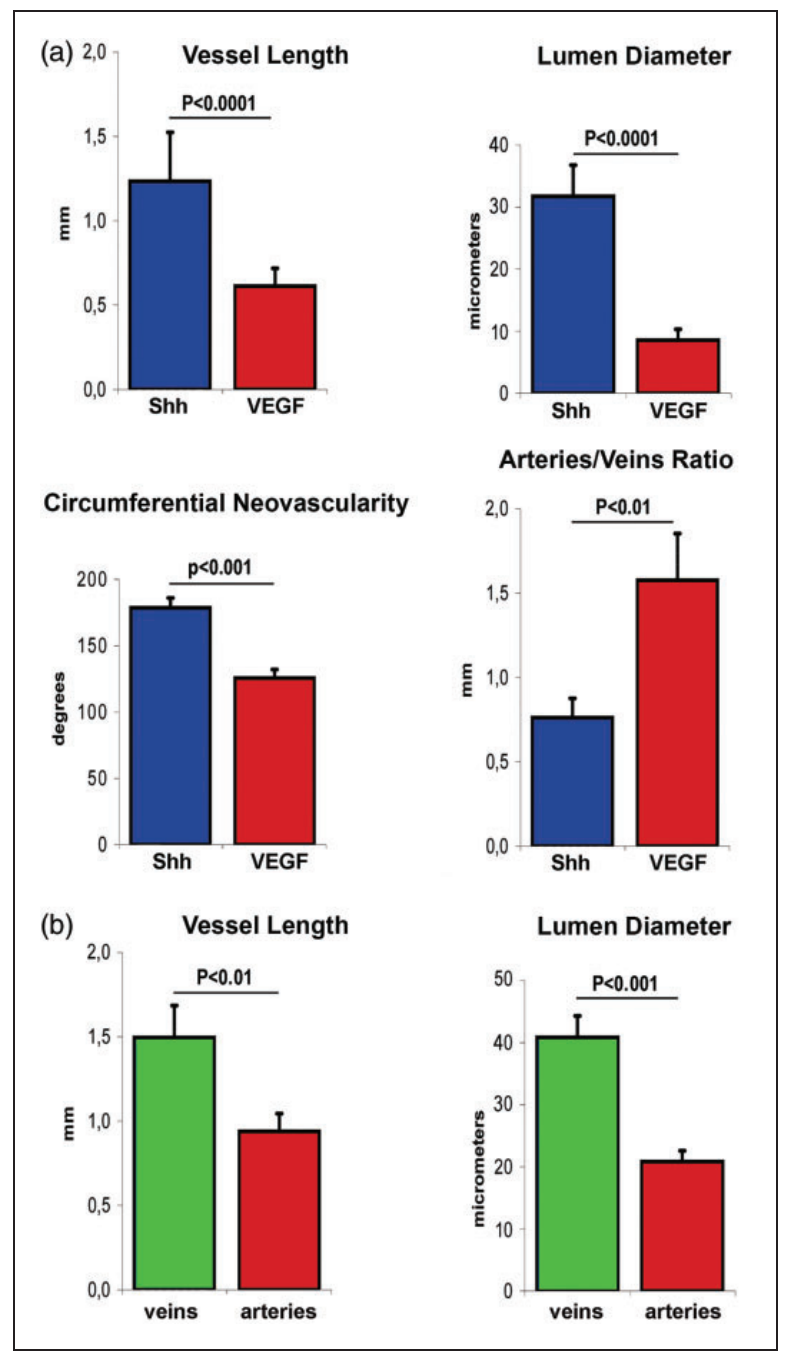

Figure 3. Quantification and characterization of Shh- and VEGF-induced corneal angiogenesis. (a) Vessel length $(\mathrm{P}<0.000 \mathrm{I})$, lumen diameter $(\mathrm{P}<0.000 \mathrm{I})$, and circumferential extent of neovascularity $(\mathrm{P}<0.00 \mathrm{I})$ are significantly greater in Shh rather than VEGF-induced corneal angiogenesis, while the ratio between arteries and veins is greater in VEGF-induced angiogenesis compared to Shh-induced angiogenesis $(\mathrm{P}<0.0 \mathrm{I})$. (b) In the setting of Shh-induced corneal angiogenesis, veins are significantly longer than arteries $(\mathrm{P}<0.0 \mathrm{I})$ and have a significantly greater lumen diameter compared to arteries $(\mathrm{P}<0.00 \mathrm{I})$. Shh: Sonic hedgehog; VEGF: vascular endothelial growth factor. 
versus $121.1 \pm 8.7$ degrees; $\mathrm{P}<0.001)$. The arteries/ veins ratio was $0.7 \pm 0.1$ and $1.6 \pm 0.3$ in Shh- and VEGF-induced angiogenesis, respectively $(\mathrm{P}<0.01)$. We also quantified mean length and diameter of arteries and veins in the context of Shh-induced angiogenesis (Figure 3(b)). Arteries and veins had a mean length of $0.9 \pm 0.1$ and $1.5 \pm 0.2 \mathrm{~mm}$, respectively $(\mathrm{P}<0.01)$. Mean lumen diameter was $20.5 \pm 2.3$ and $41.3 \pm 4.2 \mu \mathrm{m}$ for arteries and veins, respectively $(\mathrm{P}<0.001)$.

\section{Shh-induced angiogenesis has AVM-like features in the brain}

A tangle of enlarged and tortuous vessels was found at the site of injection in brain hemispheres treated with phShh, while no visible changes were found in the vasculature of contralateral brain hemispheres injected with the empty plasmid (Figure 4).

Some of these vessels were strongly positive for SMA, while others were clearly $\alpha$ SMA negative (Figure 5(a)). Such heterogeneous aSMA positivity suggests that these vessels are phenotypically different and that $\alpha$ SMA-positive vessels are more likely to be arteries, while $\alpha$ SMA-negative vessels are more likely to be veins. Digital analysis demonstrated that $41.1 \pm 11.0 \%$ of the vessels forming the vascular tangles found in the five brain hemispheres injected with the phShh were positive for $\alpha$ SMA, while the remaining vessels were $\alpha$ SMA negative (Figure 5(b)). Quantitative analyses were performed to assess the mean lumen diameter of $\alpha \mathrm{SMA}$-positive and -negative vessels: it was $67.5 \pm 14.5 \mu \mathrm{m}$ among $\alpha$ SMA-positive vessels and $70.5 \pm 15.5 \mu \mathrm{m}$ among $\alpha$ SMA-negative vessels, substantially greater than the size of surrounding brain microvasculature (Figure 5(c)).

In many histological sections, it was possible to identify $\alpha$ SMA-positive vessels directly connected to $\alpha$ SMAnegative vessels, which suggests the existence of direct arteriovenous communications in the setting of Shhinduced angiogenesis in the brain (Figure 6(a)). Interestingly, there was rarefaction of the brain vasculature in the area surrounding the neoangiogenic tangle, compared to the same brain area in the contralateral hemisphere (Figure 6(b)), suggesting that the arteriovenous structures grown in response to phShh injection might have hemodynamic effects on the surrounding brain vasculature, similar to the blood flow alterations seen in the presence of a 'steal phenomenon'.

We looked for COUP-TFII expression in the context of phShh-induced brain neovasculature, in comparison with COUP-TFII expression in the contralateral brain hemisphere. We found that the large vessels that characterize phShh-induced brain angiogenesis contain several COUP-TFII-positive cells, both at the level of the endothelial layer and the

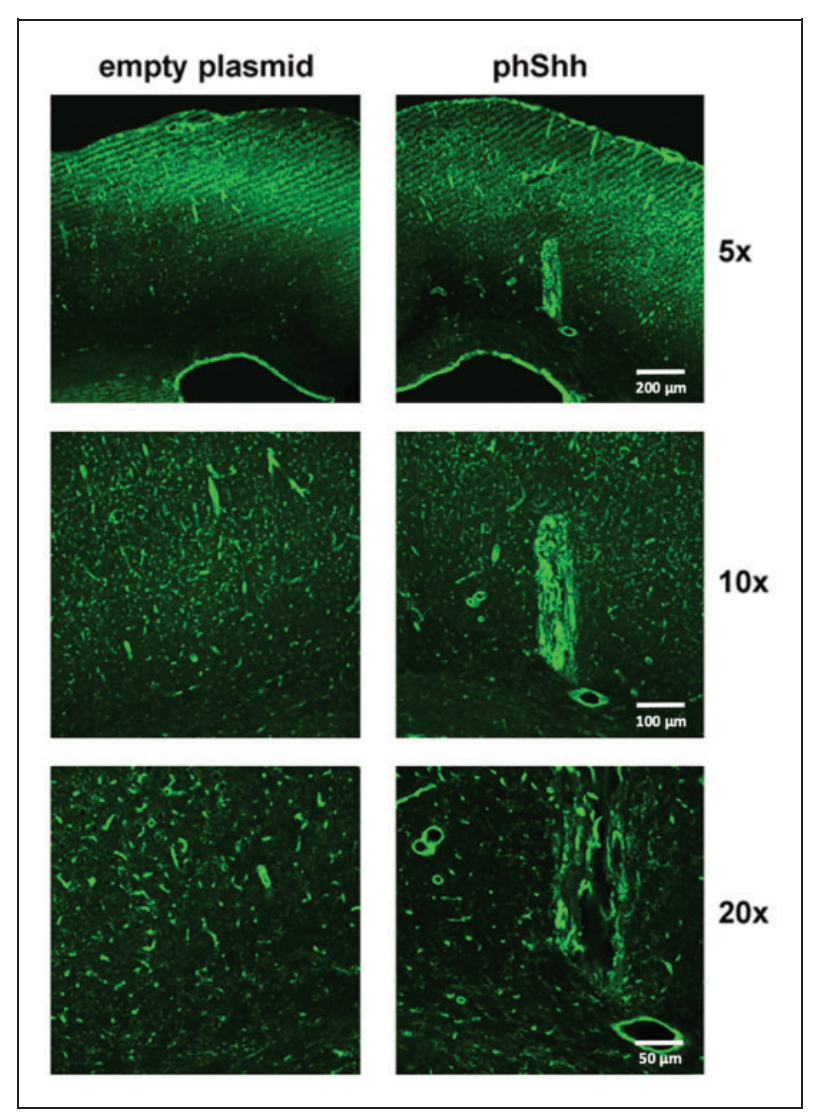

Figure 4. Effects of phShh injection in rat brain. In five rats, one brain hemisphere was treated with an intracerebral injection of phShh, while the contralateral hemisphere received an injection of empty plasmid. A robust neoangiogenic process developed in the hemispheres injected with phShh (right panels, $5 \times, 10 \times$, and $20 \times$ magnification), while no changes were observed in the vascularization of the brain hemisphere treated with the empty plasmid (left panels, $5 \times, 10 \times$, and $20 \times$ magnification).

vascular wall (Figure 7(a)). In contrast, the vasculature of the brain hemispheres that were injected with the empty plasmid was virtually negative for COUP-TFII expression (Figure 7(b)).

The histological analysis of brains injected with pCMV-DsRedCherry revealed the presence of mCherry fluorescence along the track of the needle and around the injection site. There was also mCherry-positive fluorescence within the wall layers and around vascular structures. Some mCherrypositive cells were positive for GFAP, indicating that some astrocytes had been infected by the plasmid. No mCherry-positive fluorescence was found in neurons. These results are shown in Supplementary Figure 1.

\section{Discussion}

Our study presents several new findings. First, it shows for the first time that the Shh pathway is active in 


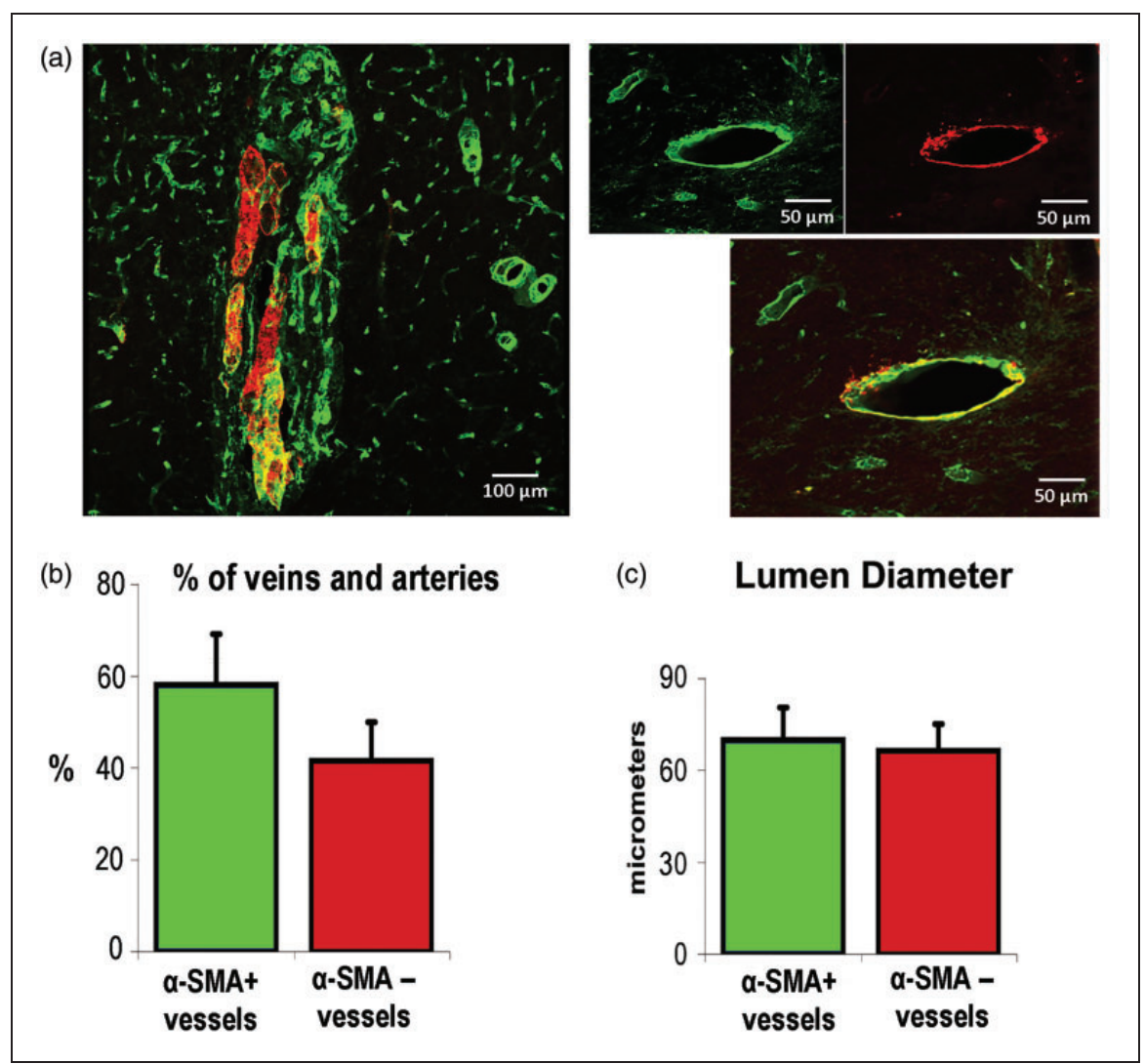

Figure 5. Presence of $\alpha S M A$-positive and $\alpha$ SMA-negative vessels in the context of Shh-induced brain neoangiogenesis. (a) Sections of brain hemispheres treated with phShh were stained for CD3I and $\alpha$ SMA. In the context of phShh-induced brain neoangiogenesis, it was possible to distinguish vessels that were $\alpha$ SMA-positive (red/yellow staining) and vessels that were $\alpha$ SMA-negative (green staining). (b) Quantification of the percentage of $\alpha$ SMA-positive and $\alpha$ SMA-negative vessels within the vascular tangles grown in response to phShh intracerebral injection. (c) Quantification of the mean lumen diameter of $\alpha$ SMA-positive and $\alpha$ SMA-negative vessels within the vascular tangles grown in response to phShh intracerebral injection.

human brain AVMs. Second, it provides evidence that Shh has the ability to stimulate the growth of an ensemble of arteries and veins in vivo, with many direct arteriovenous connections. Third, it demonstrates that intracerebral injection of Shh results in the generation of tangles of vessels that have many characteristics that are strongly reminiscent of those displayed by brain AVMs. Taken together, these data identify Shh as a novel potential player in the pathogenesis of AVMs.

Regarding our analysis of the human brain AVM samples, it is important to point out that all the specimens that we analyzed exhibited vessels that were strongly immunopositive for the Shh ligand, as well the proteins Gli1, which is the main transcription factor of the canonical Shh pathway, and COUPTFII, which is a target of the non-canonical Shh pathway, although there were many phenotypical differences among the patients from whom the AVMs were obtained, in terms of age, gender, AVM location, size of the nidus, Martin-Spetzler grading, history of bleeding, and presence of seizures (as shown in Supplementary Table 1). In contrast, the control brain specimens were all consistently negative for Shh, Gli1, and COUP-TFII expression, despite the fact that also these specimens were from phenotypically different patients who had undergone epilepsy surgery (as shown in Supplementary Table 2). The activation of the Gli1-dependent canonical pathway is known to be responsible for the upregulation of VEGF by Shh, which eventually leads to the activation of Notch and the determination of an arterial phenotype in ECs. On the other hand, the activation of COUP-TFII by Shh is known to lead to the downregulation of Notch, with the consequent determination of a venous phenotype in ECs. This is consistent with the recent demonstration that ECs of human brain AVMs co-express both arterial and venous markers, as they consist of not terminally differentiated vessels. ${ }^{2}$ While the presence of COUP-TFII in human brain AVMs has been shown previously, ${ }^{2,27}$ ours is the first demonstration that $\mathrm{Shh}$ and Glil are aberrantly expressed in the endothelial 


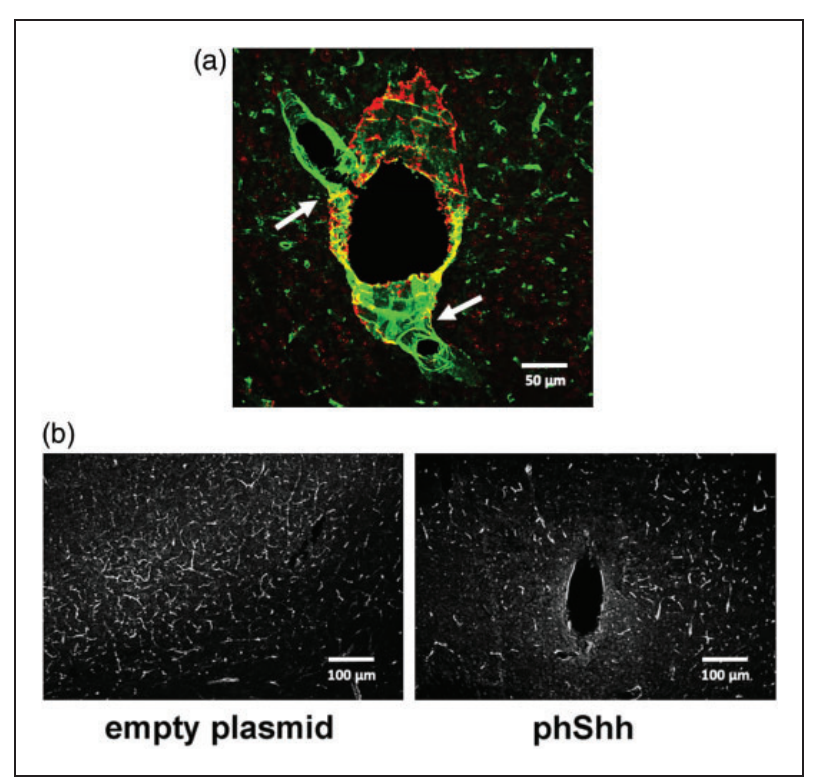

Figure 6. Presence of direct arteriovenous connections in the context of Shh-induced brain neoangiogenesis. (a)

Representative image of direct connections (white arrows) between two $\alpha$ SMA-negative vessels (green staining) and one aSMA-positive vessel (red/yellow staining) within phShh-induced brain neoangiogenesis. (b) Rarefaction of the brain vasculature in the area surrounding phShh-induced neoangiogenesis compared to the same brain area in the contralateral hemisphere injected with the empty plasmid.

layer of human brain AVMs. Indeed, there is only one report that has analyzed the expression levels of various components of the Hh pathways in brain AVMs, finding heterogeneous expression of various Hh ligands and the Hh receptor Patched-1 across the AVMs that were analyzed, without conclusive results. ${ }^{27}$ However, in such report, the $\mathrm{Hh}$ pathway was only collaterally investigated, as the study was focused on COUP-TFII and other molecules involved in venous and lymphatic signaling. In addition, the analysis of the Hh pathway was performed only by quantitative real-time polymerase chain reaction on total mRNA extracted from whole AVM specimens, without any histological examination. Since, as indicated by our results, Shh and Gli1 are specifically expressed in ECs, and ECs constitute only $1-2 \%$ of the total cells in cerebral $\mathrm{AVMs},{ }^{28}$ the analysis of total mRNA obtained from whole AVM specimens is likely to underestimate the expression levels of genes involved in $\mathrm{Hh}$ signaling in ECs. Analysis of gene expression at the single EC level, or upon isolation of ECs from human brain AVMs, would be necessary to overcome this issue.

Regarding the results of our corneal model experiments, they are consistent with our previous demonstration that Shh-induced neovasculature is different from that induced by VEGF and consists of enlarged and twisted vessels without an organized network. ${ }^{13}$ Compared to our previous publication, the novelty of the present study is the analysis of the arterial versus venous phenotype of Shh-induced corneal neovessels. Indeed, in this study, we provide evidence that Shh has the ability to stimulate the growth of an ensemble of arteries and veins in vivo, with many direct arteriovenous connections. This finding is particularly relevant because it has been replicated by us at the level of the brain. The tangles of vessels grown in response to phShh intracerebral injection have many characteristics that are strongly reminiscent of those displayed by brain AVMs: (i) vessels are tortuous and have a macroscopically visible lumen, with a bigger caliber size compared to normal cerebral microvessels; (ii) they are partly positive and partly negative for $\alpha$ SMA, which demonstrates that they are phenotypically heterogeneous; (iii) they exhibit numerous direct connections between $\alpha$ SMA-positive and $\alpha$ SMA-negative vessels, which suggests the existence of arteriovenous shunts; (iv) they contain COUP-TFII-positive cells. We are aware that $\alpha$ SMA-positivity cannot be considered a definite marker of arterial identity; however, it is a matter of fact that some of the vessels contained in Shh-induced tangles are positive for this marker, while others are negative. This indicates that these vessels do not have a homogeneous structure, but instead have important phenotypical differences, as it occurs in AVMs. In addition, there is reduced vascularization in the brain area that surrounds phShh-induced angiogenesis, reminiscent of the steal phenomenon that occurs in human brains in the presence of AVMs. This provides further support to the hypothesis that Shh-induced vascular tangles contain arteriovenous shunts. Finally, the presence of COUP-TFII-positive cells in the context of phShh-induced neovessels, and its absence in control rat brains, is consistent with the findings of previous studies that have demonstrated that COUP-TFII is aberrantly expressed in the nidus of brain AVMs in humans, while its expression is very low in the blood vessels as well as the brain parenchyma of normal tissues. ${ }^{2,25}$

The precise molecular mechanisms responsible for the formation of AVM-like structures in response to Shh injection remain to be elucidated. As outlined above, a role might be certainly played by the dual ability of Shh to regulate the expression of VEGF/ Notch and COUP-TFII, thus leading to the activation of both arterial and venous determination pathways. Nonetheless, the interplay between Hh, VEGF, Notch, and COUP-TFII during arteriovenous specification is extremely complex, and even the established view of the cascade of Hh, VEGF, and Notch acting instructively on ECs to control the choice between arterial or venous fate has been challenged, as 


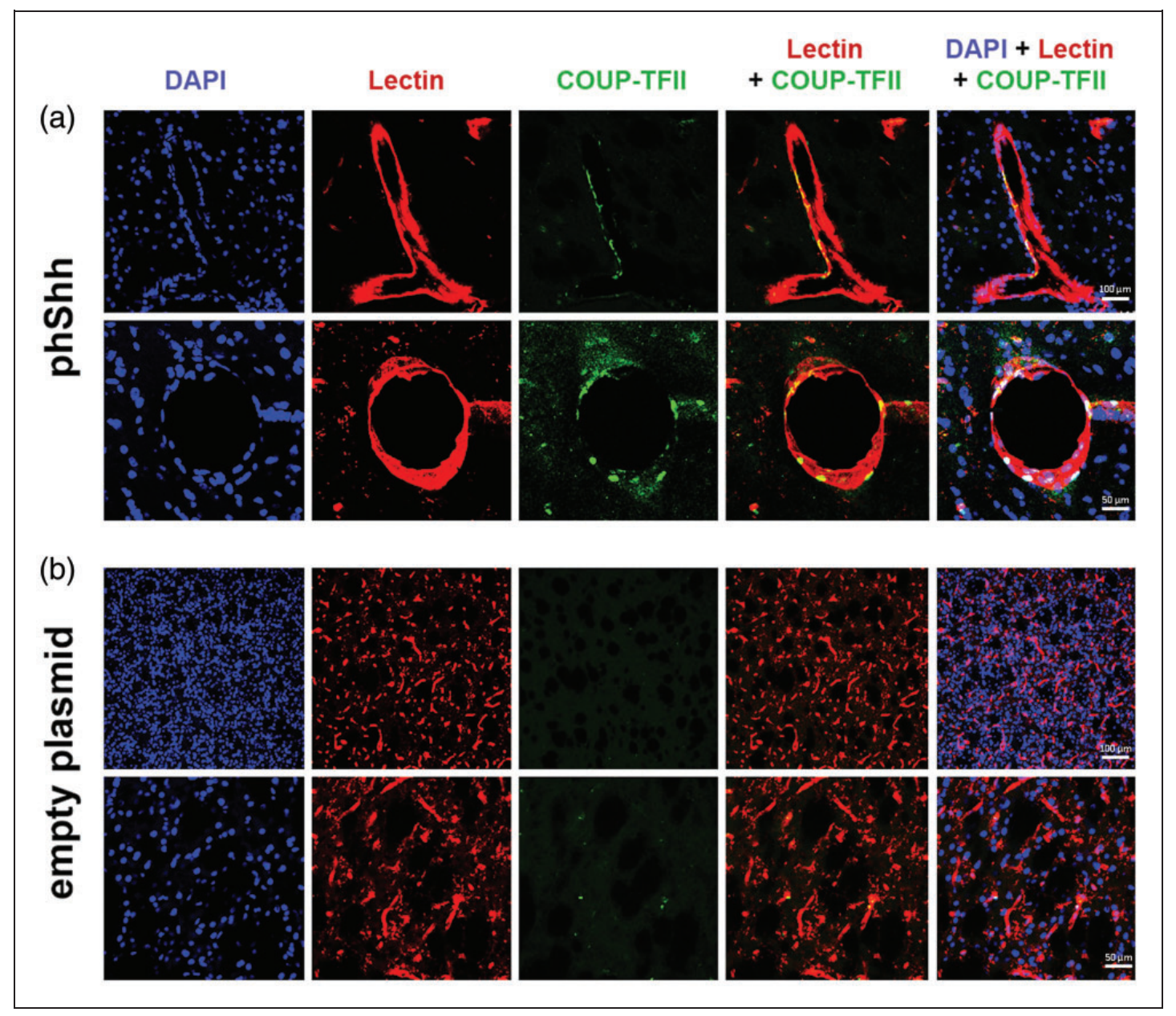

Figure 7. Expression of COUP-TFII in phShh-induced brain neovessels. Sections of brain hemispheres injected with phShh and empty plasmid were stained for DAPI (blue staining) to identify cell nuclei, lectin (red staining) to identify blood vessels, and COUPTFIl (green staining) to identify cells expressing this vascular differentiation marker. (a) COUP-TFIl-positive cells were found in the neovessels grown in the brain in response to phShh injection, both at the level of the intimal layer and the vascular wall. (b) COUPTFIl-positive cells were virtually absent in brain hemispheres injected with the empty plasmid.

demonstrated by the fact that $\mathrm{Hh}$ may bypass VEGF and use alternative pathways to induce arterial differentiation in ECs. ${ }^{29}$ What we know is that the Shh pathway is a highly preserved signal that acts upstream of many crucial angiogenic mediators, including those determining the arterial and venous identity of ECs. Also, it is important to point out that previous attempts to generate AVMs injecting angiogenic growth factors in the brain of wild-type animals have been unsuccessful. In particular, VEGF has shown the ability to generate clusters of twisted, dilated, and dysplastic microvessels with arteriovenous shunts, but only when injected in mouse models genetically predisposed to AVMs, such as the endoglin and ALK1-deficient mice. ${ }^{25,30-35}$ Different from VEGF, injection of Shh in wild-type animals has resulted in the growth of AVM-like vascular anomalies.
Finally, it remains to be determined whether and how activation of the Shh pathway may occur in the adult brain. In this context, we have already demonstrated that, in the heart and the skeletal muscle, ischemia induces strong overexpression of Shh. ${ }^{14,21}$ Additional experiments have shown that hypoxia per se - independently on ischemia - is able to induce a rapid and systemic $\mathrm{Hh}$ response in various organs of adult mice, with the $\mathrm{Hh}$ response being preceded by the accumulation of the master transcriptional regulator of hypoxia HIF1 $\alpha$ (hypoxia-inducible factor $1 \alpha$ ). ${ }^{36,37}$ There is also evidence that the Shh signaling pathway may be recapitulated in adult tissues in response to chemical and mechanical injury. ${ }^{18,24,38-40}$ Based on this, it might be speculated that the aberrant expression of Shh that we have observed in human brain AVMs might be the result of local and subclinical hypoxic, ischemic, mechanical, and/or toxic stimuli. 
Nonetheless, at the moment, no clinical evidence exists for any of these mechanisms in the pathogenesis of brain AVMs. A further hypothesis is that, once an arteriovenous shunt is formed, the commixture of arterial and venous blood may generate local hypoxia, according to the concept of the steal phenomenon, with eventual further activation of the Hh pathway.

The poor understanding of the pathogenesis of brain AVMs hinders the development of biological treatments. It has been attempted to act on molecular pathways that are disrupted in brain AVMs, including Notch and VEGF. Inhibitors of Notch have been developed and have been found to be safe in clinical trials on subjects affected by Alzheimer's disease and cancer. ${ }^{41}$ Therefore, they might represent a prospective promising therapeutic approach for brain AVMs. VEGF may be targeted using the humanized monoclonal antibody bevacizumab, which has an established safety profile and has been tested in a number of pathological conditions, including the brain cancer glioblastoma. ${ }^{42,43}$ There are also anecdotal reports on the use of bevacizumab in subjects with brain AVMs. ${ }^{44} 48$ However, efficacy and safety of this therapy in human brain AVMs remain to be determined, and a phase I trial is currently enrolling adult patients with high-grade AVMs (NCT02314377). Our findings suggest the possibility to use anti-Shh treatments as novel therapeutic approach for brain AVMs. There are $\mathrm{Hh}$ pathway inhibitors that have been approved by the Food and Drug Administration and are currently used for the treatment of basal cell carcinoma and acute promyelocytic leukemia. ${ }^{49-53}$ If our findings are confirmed and the Hh pathway is found to be a relevant player in the pathogenesis of brain AVMs, a role for anti-Hh therapies in this field could be hypothesized, with the theoretical possibility to deliver these drugs both systemically or locally, through the selective endovascular catheterization of the AVM feeders.

In conclusion, this study provides the first demonstration that the Shh pathway is active in human brain AVMs. It also shows that the angio-architecture of Shh-induced angiogenesis in vivo is strikingly reminiscent of that found in AVMs, with tortuous and dilated arteries and veins and numerous direct arteriovenous shunts. Notably, Shh has the ability to induce such aberrant angiogenic process in the brain of wild-type animals, thus in the absence of a genetic background that could facilitate the development of AVMs. Our findings might have several important implications, both at the biological, clinical, and therapeutic levels.

\section{Funding}

The author(s) disclosed receipt of the following financial support for the research, authorship, and/or publication of this article: This study was funded by a D1 grant assigned to Prof. Roberto Pola by the Università Cattolica del Sacro Cuore, Rome, Italy.

\section{Declaration of conflicting interests}

The author(s) declared no potential conflicts of interest with respect to the research, authorship, and/or publication of this article.

\section{Authors' contribution}

Igor Giarretta and Carmelo L Sturiale contributed to the concept and design, acquisition of data, analysis and interpretation of data, drafted, and revised the article; Ilaria Gatto and Simone Pacioni contributed to the acquisition, analysis, and interpretation of data; Ivana Palucci and Ilaria Gatto constructed the plasmids; Eleonora Gaetani, Angelo Porfidia, Alfredo Puca, Paolo Tondi, and Alessandro Olivi contributed to the concept and design and analysis and interpretation of data, and drafted and revised the article providing important intellectual improvements; Roberto Pallini and Roberto Pola contributed to the concept and design, acquisition of data, analysis and interpretation of data, drafted and revised the article. All the authors approved the final version of the article.

\section{ORCID iD}

Roberto Pola (D) https://orcid.org/0000-0001-5224-2931

\section{Supplemental material}

Supplemental material for this article is available online.

\section{References}

1. Nikolaev SI, Fish JE and Radovanovic I. Somatic activating KRAS mutations in arteriovenous malformations of the brain. $N$ Engl J Med 2018; 378: 1561-1562.

2. Thomas JM, Surendran S, Abraham M, et al. Gene expression analysis of nidus of cerebral arteriovenous malformations reveals vascular structures with deficient differentiation and maturation. PLoS One 2018; 13: e0198617.

3. Dalton A, Dobson G, Prasad M, et al. De novo intracerebral arteriovenous malformations and a review of the theories of their formation. Br J Neurosurg 2018; 32: 305-311.

4. Carlson TR, Yan Y, Wu X, et al. Endothelial expression of constitutively active Notch4 elicits reversible arteriovenous malformations in adult mice. Proc Natl Acad Sci USA 2005; 102: 9884-9889.

5. Murphy PA, Kim TN, Lu G, et al. Notch4 normalization reduces blood vessel size in arteriovenous malformations. Sci Transl Med 2012; 4: 117ra118.

6. Murphy PA, Lam MT, Wu X, et al. Endothelial Notch4 signaling induces hallmarks of brain arteriovenous malformations in mice. Proc Natl Acad Sci USA 2008; 105: 10901-10906. 
7. Lavine KJ, Long F, Choi K, et al. Hedgehog signaling to distinct cell types differentially regulates coronary artery and vein development. Development 2008; 135: 3161-3171.

8. Lawson ND, Vogel AM and Weinstein BM. Sonic hedgehog and vascular endothelial growth factor act upstream of the Notch pathway during arterial endothelial differentiation. Dev Cell 2002; 3: 127-136.

9. Swift MR and Weinstein BM. Arterial-venous specification during development. Circ Res 2009; 104: 576-588.

10. Salybekov AA, Salybekova AK, Pola R, et al. Sonic hedgehog signaling pathway in endothelial progenitor cell biology for vascular medicine. Int J Mol Sci 2018; 19: 3040 .

11. Krishnan V, Elberg G, Tsai MJ, et al. Identification of a novel sonic hedgehog response element in the chicken ovalbumin upstream promoter-transcription factor II promoter. Mol Endocrinol 1997; 11: 1458-1466.

12. You LR, Lin FJ, Lee CT, et al. Suppression of Notch signalling by the COUP-TFII transcription factor regulates vein identity. Nature 2005; 435: 98-104.

13. Pola R, Ling LE, Silver M, et al. The morphogen Sonic hedgehog is an indirect angiogenic agent upregulating two families of angiogenic growth factors. Nat Med 2001; 7: 706-711.

14. Pola R, Ling LE, Aprahamian TR, et al. Postnatal recapitulation of embryonic hedgehog pathway in response to skeletal muscle ischemia. Circulation 2003; 108: 479-485.

15. Shi S, Sun J, Meng Q, et al. Sonic hedgehog promotes endothelial differentiation of bone marrow mesenchymal stem cells via VEGF-D. J Thorac Dis 2018; 10: 5476-5488.

16. Guo W, Yi X, Ren F, et al. Activation of SHH signaling pathway promotes vasculogenesis in post-myocardial ischemic-reperfusion injury. Int J Clin Exp Pathol 2015; 8: 12464-12472.

17. Hayashi S, Asahara T, Masuda H, et al. Functional ephrin-B2 expression for promotive interaction between arterial and venous vessels in postnatal neovascularization. Circulation 2005; 111: 2210-2218.

18. Straface G, Aprahamian T, Flex A, et al. Sonic hedgehog regulates angiogenesis and myogenesis during post-natal skeletal muscle regeneration. J Cell Mol Med 2009; 13 : 2424-2435.

19. Biscetti F, Gaetani E, Flex A, et al. Selective activation of peroxisome proliferator-activated receptor (PPAR)alpha and PPAR gamma induces neoangiogenesis through a vascular endothelial growth factor-dependent mechanism. Diabetes 2008; 57: 1394-1404.

20. Burn SF. Detection of beta-galactosidase activity: X-gal staining. Methods Mol Biol 2012; 886: 241-250.

21. Kusano KF, Pola R, Murayama T, et al. Sonic hedgehog myocardial gene therapy: tissue repair through transient reconstitution of embryonic signaling. Nat Med 2005; 11 : 1197-1204.

22. Palladino M, Gatto I, Neri V, et al. Pleiotropic beneficial effects of sonic hedgehog gene therapy in an experimental model of peripheral limb ischemia. Mol Ther 2011; 19: 658-666.
23. Palladino M, Gatto I, Neri V, et al. Combined therapy with sonic hedgehog gene transfer and bone marrowderived endothelial progenitor cells enhances angiogenesis and myogenesis in the ischemic skeletal muscle. $J$ Vasc Res 2012; 49: 425-431.

24. Piccioni A, Gaetani E, Palladino M, et al. Sonic hedgehog gene therapy increases the ability of the dystrophic skeletal muscle to regenerate after injury. Gene Ther 2014; 21: 413-421.

25. Xu B, Wu YQ, Huey M, et al. Vascular endothelial growth factor induces abnormal microvasculature in the endoglin heterozygous mouse brain. J Cereb Blood Flow Metab 2004; 24: 237-244.

26. Marei HE, Lashen S, Farag A, et al. Human olfactory bulb neural stem cells mitigate movement disorders in a rat model of Parkinson's disease. J Cell Physiol 2015; 230: 1614-1629.

27. Shoemaker LD, Fuentes LF, Santiago SM, et al. Human brain arteriovenous malformations express lymphaticassociated genes. Ann Clin Transl Neurol 2014; 1: 982-995.

28. Hao Q, Chen XL, Ma L, et al. Procedure for the isolation of endothelial cells from human cerebral arteriovenous malformation (cAVM) tissues. Front Cell Neurosci 2018; 12: 30 .

29. Wilkinson RN, Koudijs MJ, Patient RK, et al. Hedgehog signaling via a calcitonin receptor-like receptor can induce arterial differentiation independently of VEGF signaling in zebrafish. Blood 2012; 120: 477-488.

30. Hao Q, Su H, Marchuk DA, et al. Increased tissue perfusion promotes capillary dysplasia in the ALK1deficient mouse brain following VEGF stimulation. Am J Physiol Heart Circ Physiol 2008; 295: H2250-H2256.

31. Hao Q, Zhu Y, Su H, et al. VEGF induces more severe cerebrovascular dysplasia in endoglin than in Alk1 mice. Transl Stroke Res 2010; 1: 197-201.

32. Walker EJ, Su H, Shen F, et al. Arteriovenous malformation in the adult mouse brain resembling the human disease. Ann Neurol 2011; 69: 954-962.

33. Choi EJ, Walker EJ, Shen F, et al. Minimal homozygous endothelial deletion of Eng with VEGF stimulation is sufficient to cause cerebrovascular dysplasia in the adult mouse. Cerebrovasc Dis 2012; 33: 540-547.

34. Chen W, Guo Y, Walker EJ, et al. Reduced mural cell coverage and impaired vessel integrity after angiogenic stimulation in the Alk1-deficient brain. Arterioscler Thromb Vasc Biol 2013; 33: 305-310.

35. Chen W, Sun Z, Han Z, et al. De novo cerebrovascular malformation in the adult mouse after endothelial Alk1 deletion and angiogenic stimulation. Stroke 2014; 45: 900-902.

36. Bijlsma MF, Groot AP, Oduro JP, et al. Hypoxia induces a hedgehog response mediated by HIF-1alpha. J Cell Mol Med 2009; 13: 2053-2060.

37. Spivak-Kroizman TR, Hostetter G, Posner R, et al. Hypoxia triggers hedgehog-mediated tumor-stromal interactions in pancreatic cancer. Cancer Res 2013; 73 : 3235-3247. 
38. Xu QG, Midha R, Martinez JA, et al. Facilitated sprouting in a peripheral nerve injury. Neuroscience 2008; 152 : 877-887.

39. Hashimoto M, Ishii $\mathrm{K}$, Nakamura $\mathrm{Y}$, et al. Neuroprotective effect of sonic hedgehog up-regulated in Schwann cells following sciatic nerve injury. $J$ Neurochem 2008; 107: 918-927.

40. Bond CW, Angeloni N, Harrington D, et al. Sonic Hedgehog regulates brain-derived neurotrophic factor in normal and regenerating cavernous nerves. $J$ Sex Med 2013; 10: 730-737.

41. Coric V, Salloway S, van Dyck CH, et al. Targeting prodromal Alzheimer disease with avagacestat: a randomized clinical trial. JAMA Neurol 2015; 72: 1324-1333.

42. Chinot OL, Wick W, Mason W, et al. Bevacizumab plus radiotherapy-temozolomide for newly diagnosed glioblastoma. N Engl J Med 2014; 370: 709-722.

43. Gilbert MR, Dignam JJ, Armstrong TS, et al. A randomized trial of bevacizumab for newly diagnosed glioblastoma. N Engl J Med 2014; 370: 699-708.

44. Williams BJ, Park DM and Sheehan JP. Bevacizumab used for the treatment of severe, refractory perilesional edema due to an arteriovenous malformation treated with stereotactic radiosurgery. J Neurosurg 2012; 116 : 972-977.

45. Chuang LH, Wang NK, Chen YP, et al. Mature vessel occlusion after anti-VEGF treatment in a retinal arteriovenous malformation. BMC Ophthalmol 2013; 13: 60 .
46. Preuss M, Hirsch W, Hoffmann KT, et al. Effectiveness of bevacizumab for radiation-induced cerebral necrosis in children. Pediatr Neurosurg 2013; 49: 81-85.

47. Dashti SR, Spalding A, Kadner RJ, et al. Targeted intraarterial anti-VEGF therapy for medically refractory radiation necrosis in the brain. $J$ Neurosurg Pediatr 2015; 15 : 20-25.

48. Quan K, Tang X, Song J, et al. Salvage therapy for brain arteriovenous malformations after failure of gamma knife stereotactic radiosurgery. World Neurosurg 2018; 110: e942-e951.

49. Axelson M, Liu K, Jiang X, et al. U.S. Food and Drug Administration approval: vismodegib for recurrent, locally advanced, or metastatic basal cell carcinoma. Clin Cancer Res 2013; 19: 2289-2293.

50. Khatra H, Bose C and Sinha S. Discovery of Hedgehog antagonists for cancer therapy. Curr Med Chem 2017; 24 : 2033-2058.

51. Casey D, Demko S, Shord S, et al. FDA approval summary: sonidegib for locally advanced basal cell carcinoma. Clin Cancer Res 2017; 23: 2377-2381.

52. Cohen MH, Hirschfeld S, Flamm Honig S, et al. Drug approval summaries: arsenic trioxide, tamoxifen citrate, anastrazole, paclitaxel, bexarotene. Oncologist 2001; 6: 4-11.

53. Norsworthy KJ, By K, Subramaniam S, et al. FDA approval summary: glasdegib for newly diagnosed acute myeloid leukemia. Clin Cancer Res 2019; 25: 6021-6025. 